\title{
Diabetic enteropathy — still undefeated?
}

\section{ABSTRACT}

Diabetic enteropathy is a specific diabetic complication. It could be presented as constipations, diarrhoea or faecal incontinence. The aetiology is multifactorial. The main role is attributed to irreversible autonomic neuropathy, which often coexists with peripheral sensorimotor neuropathy. Nowadays, enteropathy is diagnosed after exclusion of other causes, like coeliac disease, hypo- or hyperthyroidism, inflammatory bowel disease, small intestinal bacterial overgrowth, exocrine pancreatic insufficiency and adverse drug reaction. The article presents new reports about enteropathy reversibility after simultaneous pancreas-kidney transplantation in patients with abnormal levels of IGF-1 and IGFBP3. It provides new data on pathogenesis of enteropathy and presents new diagnostic and therapeutic approaches. (Clin Diabetol 2017; 6, 3: 105-110)

Key words: diabetes, diabetic enteropathy, diabetic autonomic neuropathy, diabetic diarrhoea, faecal incontinence

\section{Introduction}

Diabetes is a complex disease, requiring an interdisciplinary approach. It seems obvious that the diabetes care team should include an ophthalmologist, nephrologist, cardiologist and podiatrist. While it is well-known how to diagnose retinopathy, nephropathy or peripheral neuropathy, therapeutic options for enteropathy induced by diabetes include only symptomatic treatment. Diabetic enteropathy, also referred to as diabetic visceropathy, is a real problem for patients, resulting in significant decrease in the quality of life.

Address for correspondence:

Aleksandra Tomaszewska

Klinika Diabetologii i Chorób Wewnętrznych

Warszawski Uniwersytet Medyczny

e-mail: atomaszewska01@gmail.com

Translation: lek. Małgorzata Kamińska

Clinical Diabetology 2017, 6, 3, 105-110

DOI: $10.5603 /$ DK.2017.0017

Received: 08.06.2017

Accepted: 04.07.2017

\section{Symptomatology}

Diabetic enteropathy may manifest as constipation, diarrhoea and faecal incontinence. Statistically, most frequent symptom is constipation, occurring in nearly $20 \%$ of patients [1] (Tab. 1). Another common symptom is diarrhoea, which may be constant or periodic. The latter form may alternate with constipation or a normal rhythm of defecation. Most patients report symptoms occurring during the day, but some also experience night-time symptoms [1, 2]. Among patients with diarrhoea, about $60 \%$ have diabetes-related diarrhoea [3]. Faecal incontinence, isolated or accompanying diarrhoea, is undoubtedly a symptom that significantly impairs the quality of life. It is the second most frequent symptom of enteropathy. In most cases, it is associated with loose stools, which is an additional factor impairing the mechanism of normal bowel control [4].

\section{Pathogenesis of enteropathy}

Neuropathic theory

The aetiology of enteropathy is multifactorial and remains unexplained. Currently, the main role is attributed to irreversible diabetic autonomic neuropathy (DAN), which significantly disturbs regulation of the enteric autonomic system activity and, therefore, intestinal motor activity and function [1]. It is caused, among other factors, by hyperglycaemia-induced microangiopathy (Fig. 1) [5]. It results from damage caused by:

- reactive oxygen species (ROS) [6, 7];

- advanced glycation end-products (AGE) [7]; excessive activation of the polyol pathway, which promotes glucose transformation to sorbitol, and thereby contributes to changed nerve excitability and increased oxidative stress [7];

Table 1. Prevalence of symptoms of diabetic enteropathy

\begin{tabular}{lc}
\hline Symptom & Prevalence in diabetic patients \\
\hline Constipation & $18 \%[1]$ \\
Diarrhoea & $9 \%[1]$ \\
Faecal incontinence & $12 \%[4]$
\end{tabular}




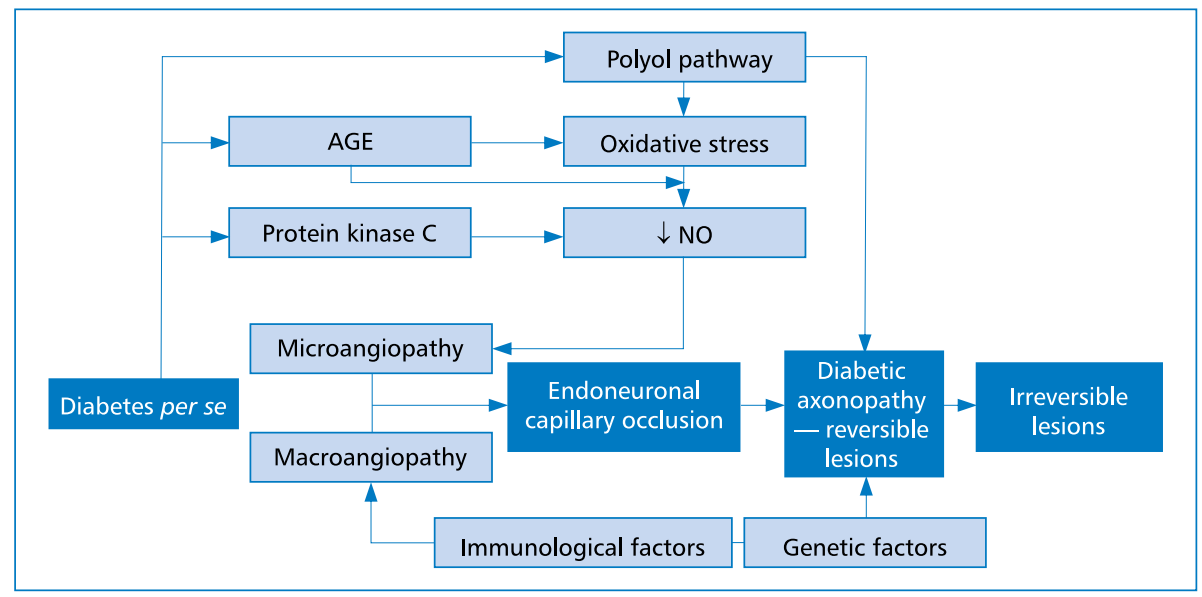

Figure 1. Pathogenesis of autonomic neuropathy — modified from [24]. AGE — advanced glycation end-products; NO — nitric oxide

- activation of diacylglycerol and protein kinase $C$ pathway, which contributes to the overproduction of proinflammatory mediators such as PG2 [8].

The neuropathic theory is confirmed by the fact that gastrointestinal symptoms in diabetic patients correlate with peripheral neuropathy [9]. Moreover, an important factor that should not be forgotten is the effect of symptomatic atherosclerosis of visceral vessels on the development of diabetic microangiopathy.

\section{Insulin-like growth factor-dependent theory}

D'Addio et al. shed new light on the problem of enteropathy, demonstrating new pathomechanisms, and arguing, contrary to previous hypotheses, that it can be reversible [10]. Researchers have shown that the serum level of an IGF-1 binding protein (IGFBP3) produced in the liver in type 1 diabetic patients with the symptoms of enteropathy was almost 5 times higher than in healthy subjects. Hepatocytes of these patients exhibit higher expression of IGFBP3 in immunohistochemistry. Moreover, IGF-1 concentrations are significantly reduced. The TMEM219 receptor has been identified in intestinal crypts. Under deficiency of IGF-1 and excess of IGFBP3, this receptor activates the caspase pathway, thereby leading to impaired differentiation of colonic stem cells (CoSC) (Fig. 2).

Histopathological examinations of intestinal mucosa of patients with enteropathy show a number of abnormalities compared to healthy individuals. In patients with enteropathy, the number of intestinal crypts is markedly reduced and they are characterized by the presence of structural abnormalities and hardening of the lamina propria of intestinal mucosa (Fig. 3). There was also a reduction in intestinal epithelial cell prolifera-

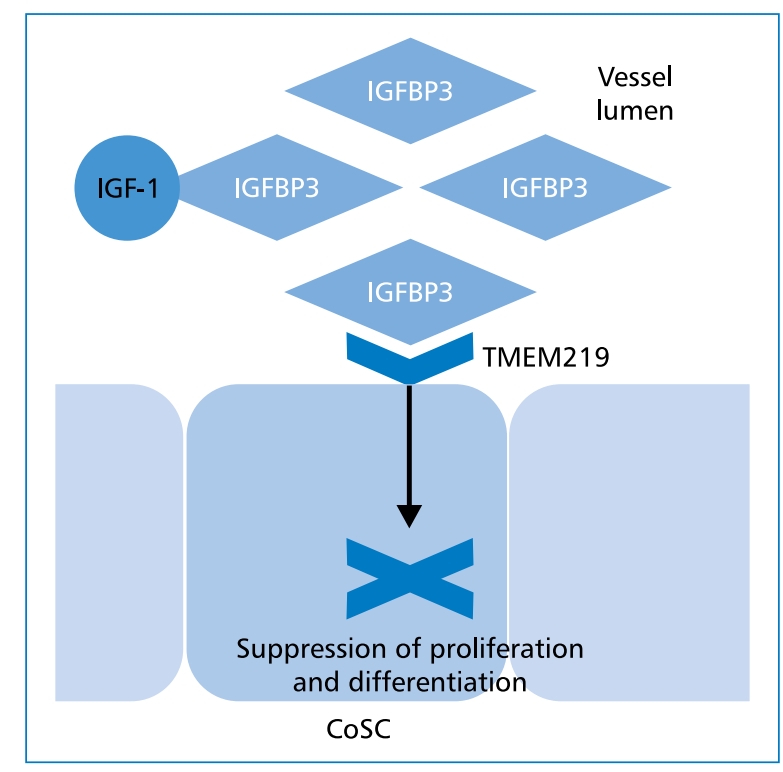

Figure 2. Mechanism of destructive action of IGFBP3 on colonic crypt stem cells IGFBP3 by binding to its receptor TMEM219 on colonic stem cell membrane suppresses proliferation and differentiation of these cells and activates apoptotic pathway. IGFBP3 - insulin-like growth factor binding protein 3; IGF-1 - insulin-like growth factor 1; CoSC - colonic stem cell; TMEM219 - transmembrane protein 219 also known as IGFBP3 receptor

tion, signs of nerve degeneration, and reduced expression of serotonin in intestinal neuroendocrine cells.

It has been demonstrated in cell culture of CoSCs from healthy individuals that isolated hyperglycaemia does not lead to direct epithelial injury, whereas the addition of serum from patients with enteropathy results in characteristic damage. Addition of excessive amount of IGFBP3 to healthy-cell culture inhibits cell proliferation even in the presence of IGF-1, but 
A

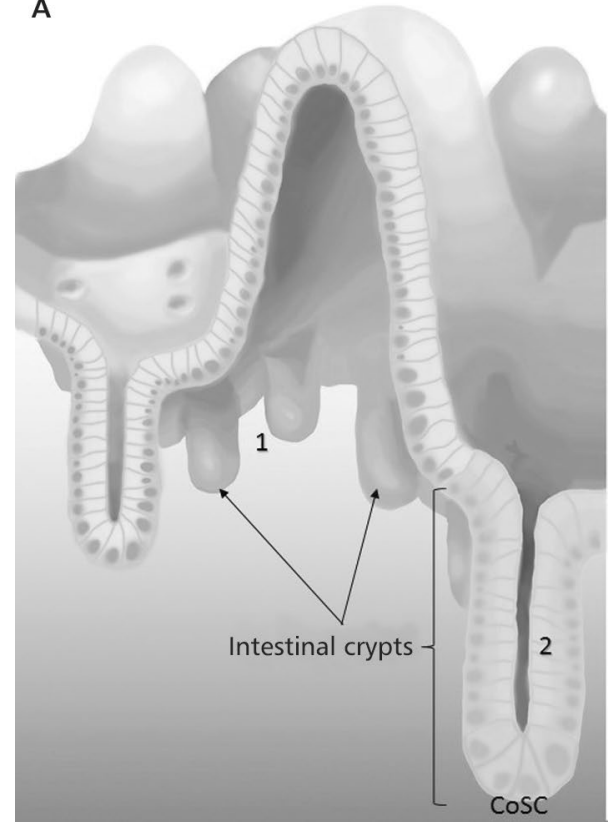

B

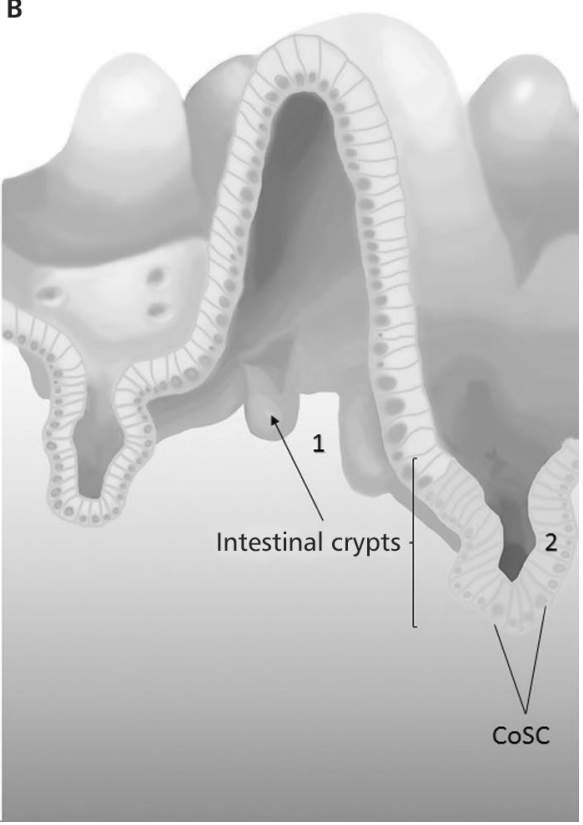

Figure 3A. Intestinal mucosa of a healthy person; B. Intestinal mucosa of a patient with diabetic enteropathy; 1 - reduced number of intestinal crypts; 2 - abnormal morphology of intestinal crypts; CoSC - colonic stem cell

this phenomenon is completely reversible after the addition of a caspase inhibitor. It seems therefore that IGFBP3 is a key factor responsible for the pathogenesis of the observed phenomenon and that by acting via the TMEM219 receptor this protein activates the apoptosis pathway independently of IGF-1. The authors are currently investigating the monoclonal antibody that blocks the TMEM219 receptor and the results are promising.

Some patients who participated in the study underwent simultaneous kidney and pancreas transplantation. During the 8-year follow-up, these patients showed significant improvement in microscopic intestinal morphology, normalization of blood IGF-1 and IGFB3 levels, and clinical improvement. Moreover, they were complete independent from exogenous insulin. This experiment fundamentally contradicts the irreversibility of enteropathy. It also allows for the conclusion indicating not only of the complexity of pathogenesis of the disease, but also the need to revise existing findings. Perhaps there are at least two forms of diabetic enteropathy: neuropathic and IGF-dependent. IGF-dependent theory is new one and certainly requires further research, but for the first time in the long while it gives hope extensive understanding of the source of the problem.

\section{Abnormalities of migrating myoelectric complex in enteropathy}

Visceropathy is associated with dysfunction of the enteric nervous system (ENS), which is a division of autonomic nervous system. Its main components are myenteric (Auerbach) and submucosal (Meissner) plexuses. This system is partly controlled by the central nervous system and partly acts independently via reflex activity [11].

Under normal conditions, the electrical activity of intestinal smooth muscles consists of slow waves, also known as the basal electrical rhythm (BER), and action potentials. Slow waves do not reach the threshold potential, so they do not cause intestinal muscle contraction, but they are very important because action potential can only arise at the peak of depolarization. The resulting potential propagates to the surrounding cells through lowresistance junction, causing muscle contraction $[2,11]$.

During the interdigestive period, a variable cyclic electrical activity called the migratory myoelectric complex (MMC) is recorded in the smooth muscles of the intestine. It arises mainly in the stomach. One MMC cycle consists of four phases and lasts about 90-120 minutes. In the first phase only slow waves are observed, thus there is no systolic action. At the second phase, individual action potentials appear, causing only moderate muscle contractions. Then, in the third phase, which lasts only 4-8 minutes, burst of action potential occur at the peak of the slow waves, which determines intensive intestinal motility. Phase four is the transition phase before a new cycle $[2,11]$.

Dooley et al. have determined that intestinal passage in patients with diabetes, autonomic neuropathy and unexplained diarrhoea is disturbed, and that this 
disturbance involves many MMC abnormalities. Most of MMC cycles had no antral component, and in the remaining phase III was absent or started in the small intestine omitting the stomach and duodenum [12]. In $25 \%$ of patients participating in the study by Dooley small intestinal bacterial overgrowth (SIBO) was diagnosed based on the hydrogen breath test and clinical improvement was obtained after antibiotic therapy. Although autonomic neuropathy may theoretically promote the occurrence of SIBO in diabetic patients by disturbing the intestinal passage, diabetes itself seems to have protective effect. In a study conducted at the Department of Internal Medicine and Diabetology at the Medical University of Poznan, patients with diabetes were almost two-fold more likely to have SIBO compared to a control group consisting of healthy and asymptomatic volunteers (41 vs. 75\%). However, the prevalence of autonomic neuropathy in the study group was not high (14.5\%) [13].

\section{Defaecation reflex disorders in diabetic enteropathy}

Changes occurring in the course of enteropathy lead also to impairment of defaecation reflex, which may manifest as a faecal incontinence. Under normal conditions, mechanoreceptors are stimulated in response to stretching the walls of the rectal bubble. As a result of the intramural and spinal reflexes (defaecation centre in the sacral spinal cord), the external anal sphincter is relaxed, the levator and muscle is contracted, and in conjunction with the intra-abdominal pressure, the faeces is excreted. A healthy person can voluntary stop this process by tightening external anal sphincter [11].

Aitchison et al. addressed the problem of faecal incontinence in people with diabetes with reference to pathophysiological aspects. Their study showed that patients with diabetes-related faecal incontinence showed decreased rectal and anal canal pressures, elevated rectal filling threshold, delayed external anal sphincter response, disturbed anal reflex (contraction of the external anal sphincter in response to irritation of the anal area) compared with diabetic patients with normal faecal continence and healthy volunteers [14].

\section{Diagnostics}

Diagnosis of enteropathy is even more complex than its pathogenesis. This is a diagnosis of exclusion. Despite the often obvious clinical symptoms and our conviction concerning the presence of this complication, we are obliged to carry out thorough differential diagnosis. Depending on the dominant symptom, such conditions as coeliac disease, thyroid dysfunction, inflammatory bowel disease, SIBO, exocrine pancreatic insufficiency, and finally the effects of the medications, should be taken into account [2]. Among them, the most common cause of chronic diarrhoea is metformin [3]. Attention should also be paid to the possibility of additional involvement of atherosclerosis in the development of visceropathy.

In most cases, endoscopy of upper and lower gastrointestinal tract and TSH level measurement are considered a primary examination. Coeliac disease can be excluded based on histopathological examination of the duodenal biopsy specimen obtained during gastroduodenoscopy along with anti-EmA (antiendomysium) and anti-tTG (anti-transgelatinase) IgA-class antibody titres determination, taking into account possible IgA deficiency. Exceptionally, the diagnosis can be made without biopsy based on characteristic symptoms, anti-tTG antibody level 10-fold higher than ULN, and positive results for HLA-DQ2 or HLA-DQ8 [15]. In SIBO diagnostics, the use of hydrogen breathing test is increasingly popular; however, in case of its limited availability, empirical antibiotic therapy and clinical observation are acceptable [2]. It may also be useful to test a stool sample for parasites.

Anorectal manometry is a valuable test in patients with faeces incontinence. It allows objectification of the problem and may be the first step towards providing a patient with a treatment. Symptomatic patients with diabetes have demonstrated an elevated threshold for the reception of sensory signals from the upper anal canal, which may be associated with delated perception of a need to defecate [14].

The optimal solution would be finding a single test or biomarker allowing the diagnosis to be quickly established without burdening the patient with numerous examinations. The search is still ongoing. One of the proposals is to test the length of the intestinal passage by scintigraphy performed after a standardized meal consisting of isotope-labelled eggs. Diagnosis of delayed passage through the small intestine may be established when after 6 hours $<40 \%$ of the total isotope activity in the small intestine accumulates in the ileocoecal region. In order to evaluate the passage through the large intestine, the acquisition is performed additionally at 24, 48 and 72 hours [16]. However, this method is cumbersome, long-lasting, expensive and associated with exposition to radiation and therefore is not widely used in diagnostics. An interesting, but expensive, alternative is the use of the wireless motility capsule (WMC), which is sensitive to $\mathrm{pH}$ and pressure changes in the gastrointestinal tract, and is therefore able to recognize when the environment changes from the small intestine to the large intestine [17]. The described methods are interesting from a research point 
Table 2. Pharmacological therapy in symptomatic treatment of diabetic enteropathy

\begin{tabular}{ll}
\hline Symptom & Medication \\
\hline Diarrhoea & Loperamide \\
& Diphenoxylate and atropine \\
& Rifaximin \\
& Amoxicillin and clavulanic acid \\
& Ciprofloxacin \\
& Metronidazole \\
& Doxycycline \\
& Lifestyle modification \\
& Lactulose \\
Constipation & Macrogol \\
& Cisapride \\
& Prucalopride \\
& Pyridostigmine \\
& Antidiarhoeal treatment (see above) \\
& Biofeedback \\
\hline
\end{tabular}

of view, but for the clinician they do not provide much information, because it is still necessary to exclude the causes of the altered passage other than diabetic enteropathy.

In conclusion, painstaking differential diagnosis remains the standard for the management of patients with the suspicion of diabetic enteropathy. This not only contributes to the delay in diagnosis, but also to the statistical underestimation of the scale of the problem.

\section{Treatment}

Due to unclear pathogenesis and lack of causative treatment, only symptomatic treatment is available for patients with visceropathy (Tab. 2).

Antidiarrhoeal medications, such as loperamide or atropine/diphenoxylate, are commonly used in the therapy of diarrhoea $[2,18]$. Empirical antibiotic therapy (e.g. rifaximin) is also permissible, if SIBO is suspected [2].

A key treatment for constipation is lifestyle modification involving proper hydration, high-fibre diet and physical exercise. Adjunctive treatment may include pharmacological agents, such as lactulose and macrogol. If these drugs are ineffective, the use of a prokinetic agent such as cisapride or a newly introduced 5-HT4 receptor agonists, prucalopride, should be considered [18]. Reports from a study on cholinesterase inhibitor in the form of orally administered pyridostigmine indicate that in patients with diabetic autonomic neuropathy it accelerates the passage through the colon, affecting the frequency of bowel movements and stool consistency, and facilitating defaecation [19].

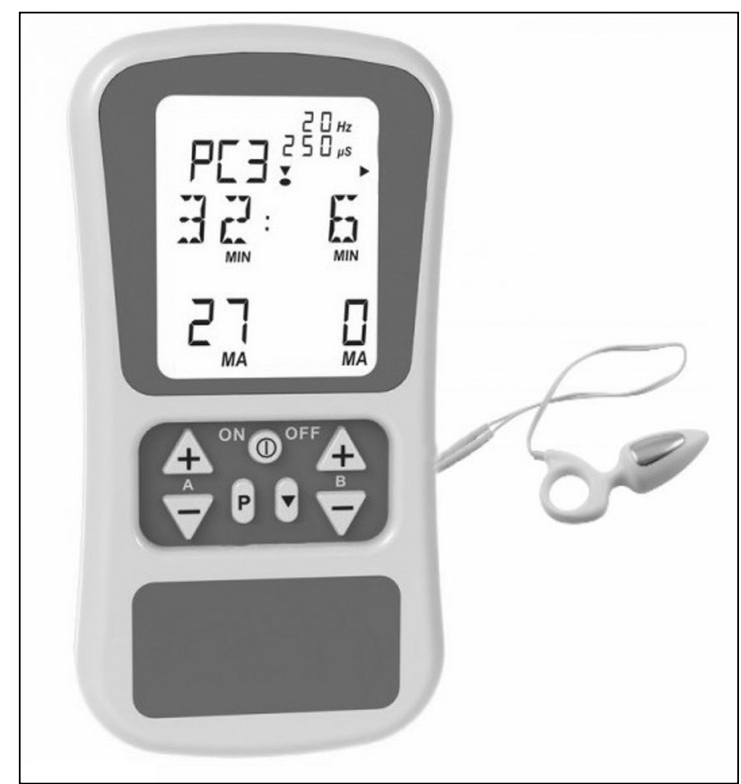

Figure 4. Electrostimulator with a rectal electrode used in biofeedback therapy for faecal incontinence

Faecal incontinence is the most difficult symptom to treat. Subsidence of diarrhoea alone may result in improvement in most patients. In refractory cases, patients may benefit from biofeedback therapy, i.e. behavioural training. Biofeedback effectively eliminates or significantly reduces the incidence of faecal incontinence in diabetic patients [20]. The patient performs anal sphincter muscle exercises at home, several times a day for 15 minutes (see Figure 4). The first effects can be seen after several months of regular exercise. The electrode located in the anal canal records the electrical activity of the anal sphincter complex during contraction. The device informs the patient if the exercise was perform correctly. Patients with sensory impairment are provided with an additional exercise program with a balloon filled with gradually increasing volume, so that the patient tightens the anal sphincter at the right time. This leads to a reflex mechanism [21]. The next step may be the use of transrectal electrostimulation, where the rectal electrode stimulates the sphincters with appropriate electric stimulus [22].

There were also attempts to treat faecal incontinence surgically by implanting magnetic anal sphincter device [23]. As previously mentioned, D'Addio et al. have reported promising results of their current studies on anti-TMEM219 monoclonal antibody (IGFBP3 receptor present in intestinal crypts), both in in vitro and in vivo and in a mouse model [10]. This gives hope for effective causal treatment in the future and can be a great alternative to symptomatic treatment that often remains ineffective. 


\section{Summary}

Diabetic enteropathy is an important clinical problem that must be addressed by a doctor who treats a diabetic patient. However, despite complex aetiopathogenesis, complicated diagnosis and lack of causative treatment, we are not completely helpless. Recent hypotheses, competing with the neuropathic theory of atrophic visceropathy, such as the destructive effect of IGFBP3 on the morphology of intestinal crypts, shed a new light on its genesis. Studies on the role of IGFBP3 and anti-TMEM219 antibodies against the IGFBP3 receptor give hope for the development of effective causative therapy and thus improvement of the quality of life. In addition, blood serum IGFBP3 can be a biomarker to significantly improve the diagnosis of diabetic enteropathy. It should be emphasized, however, that recent reports require further investigation before we can use them in clinical practice.

\section{Conflict of interest}

The authors declare no conflict of interests.

\section{REFERENCES}

1. Chandrasekharan B, Anitha M, Blatt R, et al. Colonic motor dysfunction in human diabetes is associated with enteric neuronal loss and increased oxidative stress. Neurogastroenterol Motil. 2011; 23(2): 131-138, doi: 10.1111/j.1365-2982.2010.01611.x, indexed in Pubmed: 20939847.

2. Mashimo H, May RJ, Goyal RK. Joslin's diabetes mellitus. Lippincott Williams \& Wilkins, Boston, 2005: 1069-1098.

3. Lysy J, Israeli E, Goldin E. The prevalence of chronic diarrhea among diabetic patients. Am J Gastroenterol. 1999; 94(8): 2165-2170, doi: 10.1111/j.1572-0241.1999.01289.x, indexed in Pubmed: 10445544.

4. Bytzer P, Talley NJ, Leemon M, et al. Prevalence of gastrointestinal symptoms associated with diabetes mellitus: a population-based survey of 15,000 adults. Arch Intern Med. 2001; 161(16): 1989-1996, indexed in Pubmed: 11525701.

5. Østergaard L, Finnerup NB, Terkelsen AJ, et al. The effects of capillary dysfunction on oxygen and glucose extraction in diabetic neuropathy. Diabetologia. 2015; 58(4): 666-677, doi: 10.1007/ /s00125-014-3461-z, indexed in Pubmed: 25512003.

6. Chung SSM, Ho ECM, Lam KSL, et al. Contribution of polyol pathway to diabetes-induced oxidative stress. J Am Soc Nephrol. 2003; 14(8 Suppl 3): S233-S236, indexed in Pubmed: 12874437.

7. Goldin A, Beckman JA, Schmidt AM, et al. Advanced glycation end products: sparking the development of diabetic vascular injury. Circulation. 2006; 114(6): 597-605, doi: 10.1161/CIRCULATIONAHA.106.621854, indexed in Pubmed: 16894049.

8. Koya D, King GL. Protein kinase $C$ activation and the development of diabetic complications. Diabetes. 1998; 47(6): 859-866, indexed in Pubmed: 9604860.
9. Bytzer P, Talley NJ, Hammer J, et al. Gl symptoms in diabetes mellitus are associated with both poor glycemic control and diabetic complications. Am J Gastroenterol. 2002; 97(3): 604-611, doi: 10.1111/j.1572-0241.2002.05537.x, indexed in Pubmed: 11922554.

10. D'Addio F, La Rosa S, Maestroni A, et al. Circulating IGF-I and IGFBP3 Levels Control Human Colonic Stem Cell Function and Are Disrupted in Diabetic Enteropathy. Cell Stem Cell. 2015; 17(4): 486-498, doi: 10.1016/j.stem.2015.07.010, indexed in Pubmed: 26431183.

11. Tafil-Klawe M, Klawe JJ. Wykłady z fizjologii człowieka. PZWL, Warszawa 2010: 960-990.

12. Dooley CP, el Newihi HM, Zeidler A, et al. Abnormalities of the migrating motor complex in diabetics with autonomic neuropathy and diarrhea. Scand J Gastroenterol. 1988; 23(2): 217-223, indexed in Pubmed: 3363294.

13. Adamska A, Nowak M, Piłaciński S, et al. Częstość występowania zespołu rozrostu bakteryjnego jelita cienkiego (SIBO) u pacjentów z cukrzycą. Clinical Diabetology. 2015; 4(5): 175-182, doi: 10.5603/dk.2015.0020

14. Aitchison M, Fisher BM, Carter K, et al. Impaired anal sensation and early diabetic faecal incontinence. Diabet Med. 1991; 8(10): 960-963, indexed in Pubmed: 1838049.

15. Husby S, Koletzko S, Korponay-Szabó IR, et al. ESPGHAN Working Group on Coeliac Disease Diagnosis, ESPGHAN Gastroenterology Committee, European Society for Pediatric Gastroenterology, Hepatology, and Nutrition. European Society for Pediatric Gastroenterology, Hepatology, and Nutrition guidelines for the diagnosis of coeliac disease. J Pediatr Gastroenterol Nutr. 2012; 54(1): 136-160, doi: 10.1097/MPG.0b013e31821a23d0, indexed in Pubmed: 22197856.

16. Brock C, Brock B, Pedersen AG, et al. Assessment of the cardiovascular and gastrointestinal autonomic complications of diabetes. World J Diabetes. 2016; 7(16): 321-332, doi: 10.4239/ /wjd.v7.i16.321, indexed in Pubmed: 27625746.

17. Farmer AD, Scott SM, Hobson AR. Gastrointestinal motility revisited: The wireless motility capsule. United European Gastroenterol J. 2013; 1(6): 413-421, doi: 10.1177/2050640613510161, indexed in Pubmed: 24917991.

18. Krishnan B, Babu S, Walker J, et al. Gastrointestinal complications of diabetes mellitus. World J Diabetes. 2013; 4(3): 51-63, doi: 10.4239/wjd.v4.i3.51, indexed in Pubmed: 23772273.

19. Bharucha $A E$, Low $P$, Camilleri $M$, et al. A randomised controlled study of the effect of cholinesterase inhibition on colon function in patients with diabetes mellitus and constipation. Gut. 2013; 62(5): 708-715, doi: 10.1136/gutjnl-2012-302483, indexed in Pubmed: 22677718.

20. Wald A, Tunuguntla AK. Anorectal sensorimotor dysfunction in fecal incontinence and diabetes mellitus. Modification with biofeedback therapy. N Engl J Med. 1984; 310(20): 1282-1287, doi: 10.1056/NEJM198405173102003, indexed in Pubmed: 6717494.

21. Herman RM, Wałęga P. Widera A; Biofeedback jako zachowawcza metoda leczenia nietrzymania stolca. Przegl Lek. 2004; 61: 54-59.

22. Herman RM, Wałęga P, Nowakowski M, et al. Transanal electrostimulation in the treatment of fecal incontinence following low anterior rectum resection. International Proceedings Division. 2001: 295-299.

23. Sugrue J, Lehur PA, Madoff RD, et al. Long-term Experience of Magnetic Anal Sphincter Augmentation in Patients With Fecal Incontinence. Dis Colon Rectum. 2017; 60(1): 87-95, doi: 10.1097/ /DCR.0000000000000709, indexed in Pubmed: 27926562.

24. Karnafel W, Mrozikiewicz-Rakowska B. Zespół stopy cukrzycowej. Termedia, Poznań 2010: 34. 